\title{
Use of Tolvaptan beyond Thirty Days in a Case of Refractory SIADH
}

Mamatha Siricilla', Islam Abudayyeh ${ }^{2}$ and Ala Abudayyeh ${ }^{{ }^{*}}$

${ }^{1}$ Department of General Internal Medicine \& Section of Nephrology at the University Of Texas MD Anderson Cancer Center, Texas, USA

${ }^{2}$ Department of Cardiology at Loma Linda University, Texas, USA

\section{Keywords: SIADH; Tolvaptan; Malignancy; Hyponatremia}

A 72-year-old man recently diagnosed with amyloidosis involving the mediastinum was evaluated for recurrent symptomatic hyponatremia (as low as sodium level; $106 \mathrm{mEq} / \mathrm{L}$ ) necessitating hospitalization. The patient was normotensive at presentation. His thyroid function and serum cortisol levels were normal. Laboratory findings were consistent with syndrome of inappropriate antidiuretic hormone (SIADH) with low serum osmolality $260 \mathrm{mOsm} / \mathrm{kg}$, hyponatremia, urine osmolality $366 \mathrm{mOSm} / \mathrm{kg}$ and urine sodium of $94 \mathrm{mEq} / \mathrm{L}$. On physical examination no edema was noted.

Patient was initially managed with fluid restriction, salt tablets and diuretics with no improvement in serum sodium levels. Stem cell transplant was planned for treatment of amyloidosis but was delayed because of his severe symptomatic hyponatremia. As a last resort, Tolvaptan $(7.5 \mathrm{mg})$ was started, and his serum sodium level improved to $138 \mathrm{mEq} / \mathrm{L}$ (Figure1), thus facilitating stem cell transplant. Tolvaptan was administered for approximately 60 days to achieve stable serum sodium levels and stopped thereafter.

Hyponatremia is a common electrolyte abnormality in cancer patients, and it carries a significantly negative prognosis [1]. A study by Doshi et al. [2] showed that hyponatremia is associated with longer hospital stays and higher mortality, contributing to a financial burden of $\$ 2289$ per day per admission.

Major limitations of currently available therapeutic options (eg, hypertonic saline, fluid restriction, democlocycline) are slow, unpredictable correction and renal dysfunction. Management of hyponatremia can be more challenging in the cancer setting because of the concurrent use of chemotherapeutic agents with associated hydration protocols, and coexistent renal insufficiency. Thus, there is clearly an emerging need for new oral medications with efficacy in treating hyponatremia.

Tolvaptan has been approved in the United States since 2009 for the treatment of clinically significant euvolemia and hypervolemic hyponatremia. Tolvaptan is well tolerated, and no dose adjustment is needed for renal impairment.

In a recent ADPKD (Autosomal polycystic kidney disease) trial [3], Tolvaptan delayed the progression of autosomal polycystic kidney disease using doses greater than $45 \mathrm{mg}$ /day. Three cases of severe liver injury were reported, indicating its potential for further progression to liver failure. Hence, after the aforementioned trial, the U.S. Food and Drug administration (FDA) revised the label for Tolvaptan to specify that its use not extend beyond 30 days.

Although the clinical trials for which it was primarily approved (eg, SIADH, heart failure) were of short duration [3] (i.e., less than 30 days), it was not associated with liver toxicity when administered at much lower doses.

This letter is intended to share our experience using Tolvaptan under special circumstances for a longer duration than approved by the FDA with no undue side effects and overall improvement of outcomes. We hope to encourage the use of this novel drug in a case-dependent manner.

Conflict of interest: The authors have declared that no Conflict of interest exists

\section{References}

1. Castillo JJ, Vincent M, Justice E (2012) Diagnosis and management of hyponatremia in cancer patients. The oncologist 17: 756-765.

2. Doshi SM, Shah P, Lei X, Lahoti A, Salahudeen AK (2012) Hyponatremia in hospitalized cancer patients and its impact on clinical outcomes. American Journal of kidney Diseases: the Official Journal of the National Kidney Foundation 59: 222-228.

3. Torres VE, Chapman AB, Devuyst O, Gansevoort RT, Grantham JJ, et al. (2012) Tolvaptan in patients with autosomal dominant polycystic kidney disease. The New England Journal of Medicine. 367: 2407-2418.

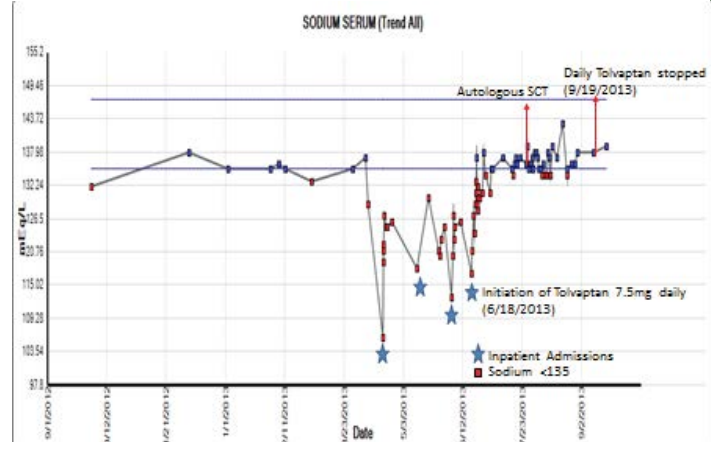

Figure 1: Sodium serum level improved to $138 \mathrm{mEq} / \mathrm{L}$.
*Corresponding author: Ala Abudayyeh M.D, Assistant Professor, MD Anderson Cancer Center, University of Texas, Division of Internal Medicine, Section of Nephrology, Unit 1468, 1400 Pressler Street, Houston, Texas 77030-3722, USA, Tel: (713)745-9331; Fax: (713)563-4491; E-mail: aabudayyeh@mdanderson.org

Received: August 08, 2015; Accepted: December 10, 2015; Published: December 17, 2015

Citation: Siricilla M, Abudayyeh I, Abudayyeh A (2015) Use of Tolvaptan beyond Thirty Days in a Case of Refractory SIADH. J Nephrol Ther 6: 229. doi:10.4172/21610959.1000229

Copyright: ( 2015 Siricilla M, et al. This is an open-access article distributed under the terms of the Creative Commons Attribution License, which permits unrestricted use, distribution, and reproduction in any medium, provided the original author and source are credited. 